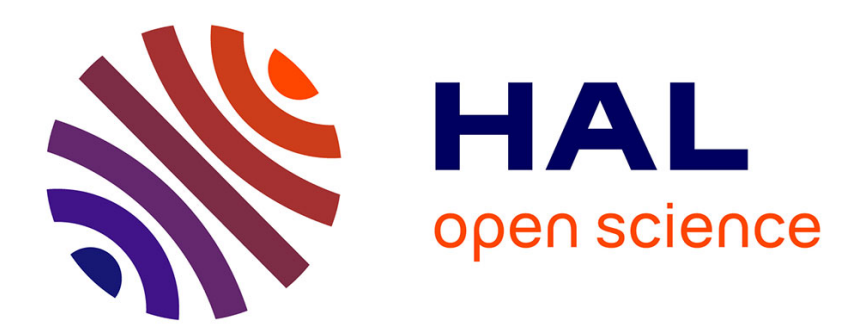

\title{
Analysis of Price Competition in a Slotted Resource Allocation Game
}

\author{
Patrick Maillé, Bruno Tuffin
}

\section{To cite this version:}

Patrick Maillé, Bruno Tuffin. Analysis of Price Competition in a Slotted Resource Allocation Game. IEEE INFOCOM'08, Apr 2008, Phoenix, United States. pp.888 - 896, 10.1109/INFOCOM.2007.141 . hal-02161968

\section{HAL Id: hal-02161968 \\ https://hal.science/hal-02161968}

Submitted on 21 Jun 2019

HAL is a multi-disciplinary open access archive for the deposit and dissemination of scientific research documents, whether they are published or not. The documents may come from teaching and research institutions in France or abroad, or from public or private research centers.
L'archive ouverte pluridisciplinaire HAL, est destinée au dépôt et à la diffusion de documents scientifiques de niveau recherche, publiés ou non, émanant des établissements d'enseignement et de recherche français ou étrangers, des laboratoires publics ou privés. 


\section{Analysis of Price Competition in a Slotted Resource Allocation Game}

\author{
Patrick Maillé \\ TELECOM Bretagne \\ 2, rue de la Châtaigneraie, CS 17607 \\ 35576 Cesson-Sévigné Cedex, FRANCE \\ Email: patrick.maille@telecom-bretagne.eu
}

\author{
Bruno Tuffin \\ IRISA/INRIA \\ Campus Universitaire de Beaulieu \\ 35042 Rennes Cedex, FRANCE \\ Email: btuffin@irisa.fr
}

\begin{abstract}
Pricing telecommunication networks has become a highly regarded topic during the last decade, in order to cope with congestion by controlling demand, or to yield proper incentives for a fair sharing of resources. On the other hand, another important factor has to be brought in: there is a rise of competition between service providers in telecommunication networks such as for instance the Internet, and the impact of this competition has to be carefully analyzed. The present paper pertains to this recent stream of works. We consider a slotted resource allocation game with several providers, each of them having a fixed capacity during each time slot, and a fixed access price. Each provider serves its demand up to its capacity, demand in excess being dropped. Total user demand is therefore split among providers according to Wardrop's principle, depending on price and loss probability. Using the characterization of the resulting equilibrium, we prove, under mild conditions, the existence and uniqueness of a Nash equilibrium in the pricing game between providers. We also show that, remarkably, this equilibrium actually corresponds to the socially optimal situation obtained when both users and providers cooperate to maximize the sum of all utilities, this even if providers have the opportunity to artificially reduce their capacity.
\end{abstract}

\section{INTRODUCTION}

The Internet, and more generally telecommunication networks, have progressively switched from an academic or monopolistic network to a commercial one with competitive service providers. In order to get a return on investment, each provider has to define a pricing strategy to charge users for the service they experience. Pricing has at the beginning been seen as a way to cope with congestion, to control demand, to deal with and satisfy heterogeneous applications with different quality of service $(\mathrm{QoS})$. It has also been regarded as a way to introduce fairness among users with respect to the traditional flat-rate pricing where light consumers pay as much as big ones. Therefore, there have been many proposals for new pricing schemes motivated by different objectives: the network planner may want to elicit users to efficiently share the scarce network resources in order to maximize social welfare (see, among others, [1]-[4]), to guarantee fairness among users [5], [6], or to maximize revenue [7]-[10]; the typical modeling tool being that of noncooperative game theory [11]. For surveys on pricing in telecommunication networks, the reader is advised to look at [12]-[14].

A very large proportion of papers deal with the monopolistic case, where there is only one provider. Though, telecommunication networks have become highly competitive and it seems primordial to us to deal with that competition in pricing models when defining the optimal prices, since competition may highly affect the results of price determination (while pricing in a monopolistic context generally means a single level of game between users, competition actually introduces an additional level of game, between providers, resulting in a so-called Stackelberg game [11]). Some typical illustrations of competition are:

- for wired access, DSL users can choose among several competing providers to connect to the Internet;

- the case of wireless access is more flexible. For example a user wishing to connect to a WiFi hotspot may be located in a zone covered by several wireless access providers, and can choose which provider to use for the time of his connection.

- The same user can/will even be able to choose between different and competitive transmission platforms: WiFi, WiMAX, 3G, Wired operators, with a possible combination of all those ones (the so-called multihoming).

Our goal in this paper is to introduce a pricing model dealing with competition. In our model, time is discretized, divided into slots, and each provider has the capability to serve a given number of packets per slot. We assume that providers do not share a common limited amount of capacity/bandwidth, but instead each provider has its own service capacity: it can model for instance competition between $3 \mathrm{G}$, WiFi and WiMAX providers for instance. Note that it does not correspond to competition at a WiFi hotspot if providers share the same bandwidth, but rather to the case where providers are being operated on different frequency channels and using different PHY modes. In our model, as soon as demand exceeds capacity at a provider, the excess demand is lost (packets are selected randomly). We consider a pricing scheme inspired by the (monopolist) one introduced in [15], where users are charged for the number of packets they submit regardless of their being treated or lost, in order to incentivize them to limit their demand. We show existence and uniqueness of a Wardrop equilibrium for demand at each provider for every combination of (fixed) prices, such that each user chooses the 
provider with the least perceived cost, where perceived cost is expressed in terms of charge and drop probability. Remark that this kind of modeling behavior is also of interest in the case of multihoming, when users are able to split their traffic between providers in order to keep the lowest overall cost. From this Wardrop equilibrium, we show, under mild conditions, that there is a unique Nash equilibrium among competing providers for the game consisting of setting prices, and we characterize that equilibrium. Those prices represent a point where no provider can increase its benefits by changing unilaterally its price. An important property is that the resulting prices correspond to the socially optimal situation, where the sum of utilities of all agents in the system -including users and providers- is maximized. This is a very desirable property, in favor of the application of such pricing strategies, since usually noncooperation leads to a loss of efficiency, quantified by the so-called price of anarchy. Another issue that we address is the interest for a provider to declare or use only a part of its real capacity. Indeed, it may happen that, due to congestion, serving less users, therefore at a higher market price, results in a larger revenue. We show here that in this context of competition, it cannot occur if demand is sufficiently elastic.

\section{A. Related work on competition}

Studying the impact of provider competition on pricing schemes is a quite new topic which is receiving increasing attention in the networking community. The importance of this field has been highlighted in [16], where it was shown that the very promising Paris Metro Pricing (PMP) scheme, which just consists in separating the network into disjoint networks served in the same manner but with different access prices, does not allow service differentiation under competition at equilibrium. In [17], a model where competitive providers play both on price and on a QoS parameter is used, and demand at each provider is driven by an arbitrary function depending on the parameters of all competitors. We feel that using a unique total demand function that is split between providers thanks to Wardrop's principle, i.e. all users choosing the provider with cheapest perceived cost, is more relevant. [18] studies competition for e-services, with also a kind of Wardrop equilibrium, but where QoS does not depend on demand. In other specific contexts, competition between wireless operators in the case of a shared spectrum (more flexible and leading to a more efficient management of the spectrum) has been studied in [19], [20]. In [19], operators are charged by a central entity for the amount of bandwidth they use, and therefore try to design proper service offers for users. Competition is shown to increase users' acceptance probability for offered service. Our analysis is based on a less specific network modeling (and without competition for capacity between providers). In [20], the authors discuss a similar competition problem, but operators only play with the power of the pilot signals of their base stations, and no pricing is considered. On the other hand, note that there is an increasing bunch of works looking at independent and selfish providers on a path, that forward traffic of competitors to ensure end-to-end delivery [21]-[23], but do not consider a direct competition for users between providers, a different perspective that we adopt.

The closest works to ours are that in [24], [25], where providers are represented by parallel links and the quality of service is delay. The price of anarchy, measuring the loss of efficiency due to competition with respect to cooperation, is determined, for fixed demand in [24] and random demand but linear delay in [25]. This is extended to the case of parallel-serial links in [26], for fixed demand. Those models consider that users are sensitive to the sum of the price charged and a congestion-dependent delay, and providers are only sensitive to their revenue. Our model is different since we assume that the total cost perceived by a user is the price charged multiplied by a congestion factor, the externality being losses here (which can be considered closer to some wireless environments). Moreover we consider that providers experience managing costs that increase with demand, those costs not being perceived by users.

Remark that the type of slotted and capacity-based model we are dealing with can be related to the one in [15], where a similar slotted capacity model is used, but with several priority traffic classes, and in the case of a monopoly instead of an oligopoly here. We therefore have a different goal: study price war between providers instead of price discrimination among users.

\section{B. Organization}

The paper is organized as follows. In Section II, we present our basic model and the required assumptions. Section III describes the socially optimal allocation when all providers cooperate, this allocation being used later on to investigate the price of anarchy for non collaborating providers. Section IV investigates the demand repartition among providers for fixed prices, following Wardrop's principle. Section V is devoted to the Nash equilibrium for the pricing game between providers; the quite long proof of the main result (existence ands uniqueness) is deferred to the appendix. Finally, in Section VI, we consider the interest for providers to artificially decrease their capacity, and provide our conclusions and directions for future research in Section VII.

\section{General MODEL}

Consider a model where time is discretized, divided into slots. Assume that there is a set $\mathcal{I}:=\{1, \ldots, I\}$ of providers in competition at an access point $(I \geq 2)$, provider $i(i \in \mathcal{I})$ having the capacity of serving $C_{i}$ packets (or units, seen as a continuous number) per slot and asking a price $p_{i}$ per packet. If demand exceeds capacity at a given provider, demand in excess is lost. We assume that lost packets are chosen uniformly over the set of submitted ones. If $d_{i}$ is the total demand at provider $i$, the number of served packets is actually $\min \left(d_{i}, C_{i}\right)$, meaning that packets are actually served with probability $\min \left(C_{i} / d_{i}, 1\right)$.

Users are assumed to be charged for each submitted packet instead of each served one in order to incentivize them to limit 
demand. The average perceived price per served traffic unit at provider $i$ is therefore

$$
\underline{p}=p_{i} / \min \left(C_{i} / d_{i}, 1\right)=p_{i} \max \left(d_{i} / C_{i}, 1\right) .
$$

Indeed, the total income of provider $i$ is $d_{i} p_{i}$ and the total service "rate" is $d_{i} \min \left(C_{i} / d_{i}, 1\right)$, giving the unit perceived price just above by a direct ratio. In that situation, it can be seen that the negative externality of congestion is expressed in terms of losses experienced by users.

We assume that total user demand is a function $D(\cdot)$ of the perceived price $\underline{p}$, and that $D$ is continuous and strictly decreasing with $\underline{p}$ on its support $\left[0, p_{\max }\right)$, with eventually $p_{\max }=+\infty$. We moreover assume that $D(0)>\sum_{i \in \mathcal{I}} C_{i}$, i.e. there is some congestion: the total resource available is not sufficient to satisfy the maximum demand level. Remark that the demand function can be interpreted in two ways (or as a combination of those two effects):

- it can stem from user heterogeneity in their willingnessto-pay for the service: consider a continuum of infinitesimally small users with fixed demand, whose willingnessto-pay (in terms of unit price) is distributed according to a given distribution. Then for a given unit price $p \geq 0$, $D(p)$ is the amount of flow generated by users with valuation larger than $p$ and is naturally decreasing.

- Likewise, the decreasingness of the total demand function $D$ can also stem from the decreasingness of individual functions.

We can also define the function $v: q \mapsto \inf \{p: D(p) \leq q\}$ (with the convention $\inf \emptyset=0$ ). Since $D$ is continuous, strictly decreasing for $p<p_{\max }$ and null for $p \geq p_{\max }$ for a given $p_{\max }$ then we simply have

$$
v(q)= \begin{cases}D^{-1}(q) & \text { if } q \in(0, D(0)) \\ p_{\max } & \text { if } q=0 \\ 0 & \text { if } q \geq D(0) .\end{cases}
$$

The quantity $v(q)$ is then the unit price that one has to impose on users in order to ensure that total demand will be $q$ (for $q \leq$ $D(0)$ ). The function $v$ is called marginal valuation function: $v(q)$ is indeed the maximum unit price that can be charged for the $q^{\text {th }}$ unit of demand without making the demand decrease: it is a nonincreasing function since $D$ is nonincreasing.

We finally define $V(q)$ as the sum of the marginal valuations of the $q$ units of users with largest willingness-to-pay, i.e.

$$
V(q):=\int_{x=0}^{q} v(x) d x .
$$

As an example, if all users experience a fixed unit price $p$, then only the $q=D(p)$ units with valuation larger that $p$ will subscribe to the service, and the sum of their marginal valuations will be $V(q)$. We refer to this function $V$ as the valuation function: $V(q)$ corresponds to the total price that the $q$ units of demand with highest marginal valuation are willing to pay to be served (remark that the function $V$ is nondecreasing and concave). For a fixed unit price $p$, the user surplus then equals $V(D(p))-p D(p)$.
A first step of our work will be to study how, for fixed prices $p_{i}(i \in \mathcal{I})$, total demand is split among providers. This is described and characterized in Section IV in terms of a Wardrop equilibrium.

Knowing a priori the distribution $\mathbf{d}:=\left(d_{1}, \ldots, d_{I}\right)$ of demand that will result from a price profile, the goal of each provider is, by playing on its unit price $p_{i}$, to maximize its net benefit

$$
R_{i}\left(p_{1}, \ldots p_{I}\right):=p_{i} d_{i}-\ell_{i}\left(d_{i}\right),
$$

where $p_{i} d_{i}$ is the money earned directly from demand, and $\ell_{i}\left(d_{i}\right)$ represents the cost for provider $i$ of managing a demand level $d_{i}$. We assume that for all $i, \ell_{i}$ is nondecreasing, differentiable and convex. Notice that contrary to the model considered in [24], this cost is not reflected here in the quality of service experienced by users, but is only perceived by providers.

The price chosen by a provider has an impact on demands, and therefore on benefits, of other providers; consequently our model induces a game between providers. Since we consider competitive providers, the framework is that of noncooperative game theory. We are going to investigate in Section V, under mild conditions, the existence and uniqueness of a Nash equilibrium for the price game, that is a price vector $\mathbf{p}:=\left(p_{1}, \ldots, p_{I}\right)$ such that no provider can increase its own benefit by unilaterally changing its access price, i.e., $\forall i \in \mathcal{I}$, $\forall p \geq 0$,

$$
R_{i}\left(p_{1}, \ldots, p_{I}\right) \geq R_{i}\left(p_{1}, \ldots, p_{i-1}, p, p_{i+1}, \ldots, p_{I}\right) .
$$

Most of our results are valid under the following assumption.

Assumption A: The marginal cost of every provider when its demand equals its capacity is lower than the global marginal valuation of the sum of all provider capacities. In other terms,

$$
\forall i \in \mathcal{I}, \quad \ell_{i}^{\prime}\left(C_{i}\right) \leq v\left(\sum_{j \in \mathcal{I}} C_{j}\right),
$$

or equivalently

$$
\forall i \in \mathcal{I}, \quad D\left(\ell_{i}^{\prime}\left(C_{i}\right)\right) \geq \sum_{j \in \mathcal{I}} C_{j},
$$

where $\ell_{i}^{\prime}$ is the derivative function of $\ell_{i}$.

We will sometimes need a slightly more restrictive assumption.

Assumption B: For each provider $i \in \mathcal{I}$, the following inequality holds:

$$
\ell_{i}^{\prime}\left(C_{i}\right) \leq\left(1-\frac{C_{i}}{\sum_{j \neq i} C_{j}}\right) v\left(\sum_{j \in \mathcal{I}} C_{j}\right) .
$$

With respect to Assumption A, Assumption B is more restrictive especially if a provider has a significant "power" in terms of capacity, i.e. if $\frac{C_{i}}{\sum_{j \neq i} C_{j}}$ is not negligible. In the extreme case of a large number of providers with the same capacity $C_{i}=C$, Assumption $\mathrm{B}$ simply resumes to 
Assumption A. In general, it is reasonable to consider that the managing costs are small, if not negligible with respect to the price users are willing to pay for the service. Therefore we expect Assumption B to hold in networks where competition occurs.

\section{Socially optimal Situation}

In this section, we define social welfare as the sum of the utilities of all agents in the system (i.e. users and providers), and study the maximal value of that criterion. It is well-known in Game Theory [27] that agent selfishness does not lead in general to a socially efficient situation. The loss of efficiency due to the divergence of user interests, often referred to as the Price of Anarchy [28], is an interesting performance measure for a game: if social welfare at an equilibrium of the game is close to its maximal value, then letting agents choose their actions selfishly can be preferable to introducing costly control or incentive schemes.

Definition 1: For a demand configuration $\mathbf{d}:=\left(d_{1}, \ldots, d_{I}\right)$, we call social welfare the quantity

$$
\mathrm{SW}:=V\left(\sum_{i \in \mathcal{I}} \min \left(d_{i}, C_{i}\right)\right)-\sum_{i \in \mathcal{I}} \ell_{i}\left(d_{i}\right) .
$$

Social welfare SW accounts for the utilities of users (the quantity $\sum_{i \in \mathcal{I}} \min \left(d_{i}, C_{i}\right)$ is the total effective user rate, and the first term of (3) is total user valuation) and of providers (the second term accounts for the costs associated to the demand configuration). Remark that no monetary exchanges appear in (3): this is due to the fact that such exchanges would be added to the providers utility and subtracted from the users utility, which would not affect the sum of all agent utilities.

We compute here the maximal value of social welfare, that will be used as a reference in the next sections.

Proposition 1: Under assumption A, the maximum value of social welfare is reached when $d_{i}=C_{i}$ for each provider $i$.

Proof: The social welfare maximization problem is expressed by

$$
\max _{\mathbf{d} \in \mathbb{R}_{+}^{I}} V\left(\sum_{i \in \mathcal{I}} \min \left(d_{i}, C_{i}\right)\right)-\sum_{i \in \mathcal{I}} \ell_{i}\left(d_{i}\right) .
$$

Notice that since $\ell_{i}$ is strictly increasing for all $i$, the objective function is strictly decreasing in $d_{i}$ for all $i \in \mathcal{I}$ when $d_{i} \geq C_{i}$, therefore our optimization problem is equivalent to

$$
\max _{\substack{\mathbf{d} \in \mathbb{R}_{+}^{I} \\ \text { subject to }}} V\left(\sum_{i \in \mathcal{I}} d_{i}\right)-\sum_{i \in \mathcal{I}} \ell_{i}\left(d_{i}\right)
$$

Since $V$ is a concave function and the provider cost functions $\left(\ell_{i}\right)_{i \in \mathcal{I}}$ are convex, this last problem is a classical convex problem (maximization of a concave function over a convex set), that can be solved by the Lagrangian method. Denoting by $\lambda_{i} \geq 0$ the Lagrange multiplier relative to the constraint $d_{i} \leq C_{i}$, the first order conditions imply that for a demand configuration $\left(d_{1}^{*}, \ldots, d_{I}^{*}\right)$ maximizing social welfare, we have

$$
\forall i \in \mathcal{I}, \quad \lambda_{i}+\ell_{i}^{\prime}\left(d_{i}^{*}\right)=p^{*},
$$

with $p^{*}:=v\left(\sum_{i \in \mathcal{I}} d_{i}^{*}\right)$ (i.e. $\sum_{i \in \mathcal{I}} d_{i}^{*}=D\left(p^{*}\right)$ ). Then the complementary slackness conditions yield

$$
\forall i \in \mathcal{I}, \quad \min \left(\lambda_{i}, C_{i}-d_{i}^{*}\right)=0 .
$$

Let us define $\mathcal{I}_{0}:=\left\{i \in \mathcal{I}: d_{i}^{*}=C_{i}\right\}$ and $\mathcal{I}_{u}:=\{i \in \mathcal{I}$ : $\left.d_{i}^{*}<C_{i}\right\}$, and assume that $\mathcal{I}_{u} \neq \emptyset$. Then for an $i_{0} \in \mathcal{I}_{u}$, (6) implies that $\lambda_{i_{0}}=0$, and we have

$$
\begin{aligned}
\sum_{i \in \mathcal{I}} C_{i} & >\sum_{i \in \mathcal{I}_{u}} d_{i}^{*}+\sum_{i \in \mathcal{I}_{0}} C_{i}=\sum_{i \in \mathcal{I}} d_{i}^{*}=D\left(p^{*}\right) \\
& =D\left(\ell_{i_{0}}^{\prime}\left(d_{i_{0}}^{*}\right)\right) \\
& \geq D\left(\ell_{i_{0}}^{\prime}\left(C_{i_{0}}\right)\right) \\
& \geq \sum_{i \in \mathcal{I}} C_{i},
\end{aligned}
$$

where the second line comes from (5), the third one from the convexity of $\ell_{i_{0}}$ and the nonincreasingness of $D$, and the last line stems from Assumption A. We reach a contradiction, which means that $\mathcal{I}_{u}=\emptyset$, and establishes the proposition.

\section{WARDROP EQUILIBRIUM FOR USERS}

In this section, we investigate how demand is split among providers when the price $p_{i}$ per unit of sent traffic is fixed by each provider $i \in \mathcal{I}$. We assume that users are infinitely small and therefore their choices do not individually affect the demand levels (and therefore the perceived costs) of the different providers: such users are said to be price-takers since they individually have no influence on prices. The outcome resulting from such user interactions is described by Wardrop's principle [29]: demand is distributed in such a way that all users choose one of the cheapest providers. As a result, the user perceived price is the same for all providers with positive demand, and is lower than the unit price $p_{i}$ of providers $i$ with no demand. Since we considered the case of elastic demand, the total demand level must also correspond to the common perceived price on all providers that receive some demand. Those properties are summarized in the next definition.

Definition 2: For given capacity $\mathbf{C}:=\left(C_{1}, \ldots, C_{I}\right)$ and price $\mathbf{p}=\left(p_{1}, \ldots, p_{I}\right)$ configurations, a user equilibrium is a demand configuration $\mathbf{d}=\left(d_{1}, \ldots, d_{I}\right)$ such that for all $i, j \in \mathcal{I}$,

$$
\begin{aligned}
& d_{i}>0 \quad \Rightarrow \quad p_{i} \max \left(1, d_{i} / C_{i}\right) \leq p_{j} \max \left(1, d_{j} / C_{j}\right), \\
& d_{i}>0 \quad \Rightarrow \quad p_{i} \max \left(1, d_{i} / C_{i}\right)=v\left(\sum_{k \in \mathcal{I}} d_{k}\right) .
\end{aligned}
$$

According to Condition (7), all providers with positive demand have the same perceived unit price, otherwise part of the demand will have interest in changing providers. Condition (8) states that total demand corresponds to that common value of the perceived unit price via the demand function $D$.

Remark that this definition corresponds to the one provided in [25], but for a different pricing scheme and different cost functions. The following proposition characterizes the user equilibria corresponding to fixed capacities and prices. 
Proposition 2: For any capacity and price configuration, there exist a (possibly not unique) user equilibrium demand configuration.

Moreover, at a user equilibrium $\mathbf{d}$, the common perceived unit price $\underline{p}$ of providers $i$ with $d_{i}>0$ is unique and equals

$$
\begin{gathered}
\underline{p}=\inf \left\{p: D(p) \leq \sum_{i \in \mathcal{I}} f_{i}(p)\right\}, \\
\text { where } f_{i}(p):=\mathbb{1}_{\left\{p \geq p_{i}\right\}} \frac{C_{i}}{p_{i}} p .
\end{gathered}
$$

Proof: Since the perceived unit price functions for each provider are continuous and nondecreasing, for any value of the total demand $d$ there exist a demand configuration $\mathbf{d}$ such that $\sum_{i} d_{i}=d$ and condition (7) is satisfied. Moreover the common value $p_{c}(d)$ of the perceived unit price at providers with strictly positive demand is unique when total demand is fixed (this was established in [30]) when perceived unit prices are continuous and nondecreasing functions of demand. It is easy to see that $p_{c}(d)$ is a continuous and nondecreasing function of total demand $d$.

The second property, given by condition (8), that a user equilibrium must satisfy can be expressed as $p_{c}(d)=v(d)$. Since $v$ is a continuous nonincreasing function and $p_{c}$ is continuous, nondecreasing and tends to infinity as $d$ becomes large $\left(p_{c}(0)=\min _{i} p_{i}\right.$ and for $d$ sufficiently large $p_{c}$ is a strictly increasing linear function of $d$ ), then

- if $v(0) \geq \min _{i} p_{i}$ then there exists a $\underline{d}$ such that $p_{c}(\underline{d})=$ $v(\underline{d})$. The corresponding value $p:=v(\underline{d})$ is unique due to the respective monotonicity properties of $p_{c}$ and $v$.

We now prove that $\underline{p}$ verifies (9). Since we assumed that $v(0)=p_{\max } \geq \min _{i} p_{i}$, we necessarily have $\underline{d}=D(\underline{p})$ from (1). Moreover (7) implies that $d_{i}=f_{i}(\underline{p})$, so $D(\underline{p})=\sum_{i \in \mathcal{I}} f_{i}(\underline{p})$. Relation (9) then comes from $p \leq p_{\max }$ and the strict decreasingness of $D$ on $\left(0, p_{\max }\right)$.

- if $v(0)<\min _{i} p_{i}$ then there is no intersection point between $p_{c}$ and $v$, since no user is interested in subscribing to the service at a unit price $\min _{i} p_{i}$. Therefore the configuration $\mathbf{d}=(0, \ldots, 0)$ is the unique user equilibrium.

Figure 1 displays the demand function $D$ and the function $\sum_{i \in \mathcal{I}} f_{i}$ for a given price configuration, and illustrates the existence and uniqueness of the Wardrop equilibrium perceived price $p$. Function $f_{i}$ basically represents the corresponding share of demand ${ }^{1}$ that provider $i$ can get at a given perceived price $p$.

Remark 1: From this proposition, we are able to characterize the unique perceived price $\underline{p}$. Total demand is therefore $D(\underline{p})$. For all providers with price $p_{i} \neq \underline{p}$, demand $d_{i}$ is then $d_{i}=f_{i}(p)$. All providers such that $p_{i}=p$ (if any) share the remaining demand $D(\underline{p})-\sum_{i: p_{i}<p} d_{i}$, all possible sharing providing a Wardrop equilibrium. In this sense, there

\footnotetext{
${ }^{1} f_{i}$ is indeed the generalized inverse of the perceived unit price function $d_{i} \mapsto p_{i} \max \left(1, d_{i} / C_{i}\right)$, i.e. $f_{i}(p)=\sup \left\{d_{i}: p_{i} \max \left(1, d_{i} / C_{i}\right) \leq p\right\}$.
}

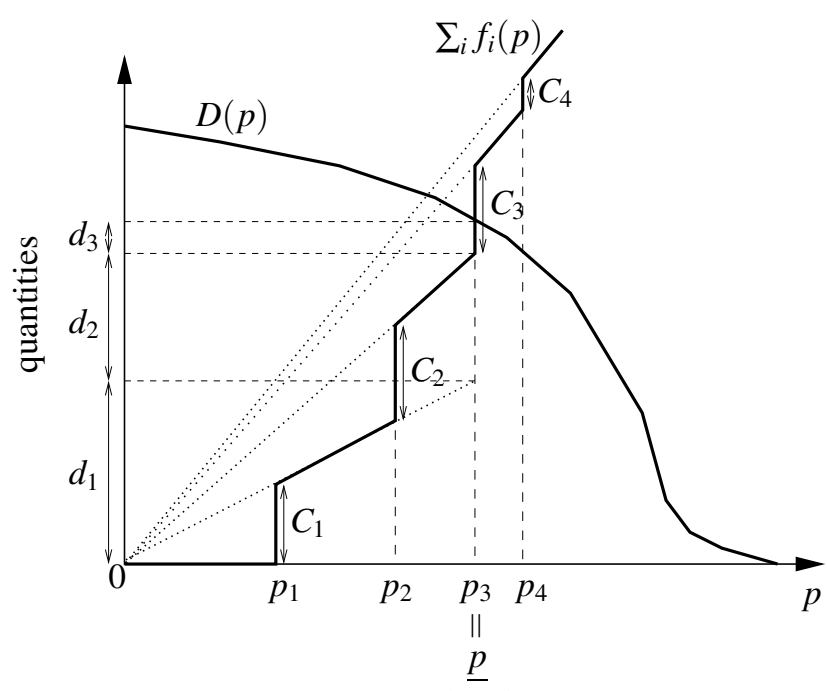

unit price

Fig. 1. Wardrop equilibrium for three providers and a given price configuration: the common perceived price at each provider with positive demand (i.e. providers $1,2,3$ ) is $\underline{p}=p_{3}$.

is not always uniqueness for the Wardrop equilibrium and the corresponding revenues for each provider are not necessarily unique. Note nonetheless that the resulting total revenue is always the same. Moreover, we will see in the following that when providers are at a Nash equilibrium of the pricing game, then the corresponding user Wardrop equilibrium is unique.

\section{PRICE COMPETITION AMONG PROVIDERS}

In this paper, we consider that providers setting their prices is the first stage of a two-level game, where the second stage corresponds to users reacting according to the Wardrop equilibrium described in Definition 2. We assume that providers are aware of their advantage of playing first, i.e. they take into account users' reaction when determining their price. This common knowledge complicates the competition among providers, and is the purpose of the analysis in this section.

Before analysing the pricing game between providers, we first prove a lemma that establishes some monotonicity results of Wardrop equilibria with respect to the price configuration p. In the following, we often compare two situations from the point of view of one provider to study the consequences of its price decisions. We therefore use the superscript " $n$ " to refer to the values (price, demand, benefit) corresponding to a new situation in contrast to the reference situation.

Lemma 1: Consider a price configuration $\mathbf{p}=\left(p_{1}, \ldots, p_{I}\right)$, and $i \in \mathcal{I}$. If provider $i$ raises its price, i.e. chooses $p_{i}^{n}>p_{i}$ while all other providers $j \neq i$ keep their price to $p_{j}$, then

- the common perceived price (for providers with positive demand) increases: $p^{n} \geq p$,

- if $d_{i}>0$ then the demand of provider $i$ strictly decreases: $d_{i}^{n}<d_{i}$.

Proof: Since $p_{i}^{n}>p_{i}$ then from (10) we have the functional inequality $f_{i}^{n} \leq f_{i}$. Therefore, (9) implies that for 
all $\varepsilon, 0<\varepsilon \leq \underline{p}$,

$D(\underline{p}-\varepsilon)>D(\underline{p}) \geq \sum_{j \in \mathcal{I}} f_{j}(\underline{p}) \geq \sum_{j \in \mathcal{I}} f_{j}(\underline{p}-\varepsilon) \geq \sum_{j \in \mathcal{I}} f_{j}^{n}(\underline{p}-\varepsilon)$,

which yields $\underline{p}^{n} \geq \underline{p}$.

Now assume that $d_{i}>0$ and that $d_{i}^{n} \geq d_{i}$. The equality $\underline{p}=p_{i} \max \left(d_{i} / C_{i}, 1\right)$ implies that $\underline{p}^{n}>\underline{p}$. Therefore the demand on all links $j \neq i$ should strictly increase to reach the same perceived price $p^{n}$. This would mean that total demand increases, which is in contradiction with $\underline{p}^{n}>\underline{p}$ and the nonincreasingness of total demand $D$.

We can now establish our main result.

Proposition 3: Assume that $\forall i \in \mathcal{I}, \ell_{i}$ is strictly increasing and convex. Under Assumption B, there exists a unique Nash equilibrium on price war among providers, given by

$$
\forall i \in \mathcal{I}, \quad\left\{\begin{array}{l}
d_{i}=C_{i} \\
p_{i}=p^{*}
\end{array},\right.
$$

where $p^{*}=v\left(\sum_{j \in \mathcal{I}} C_{j}\right)$, that is

$$
\sum_{i \in \mathcal{I}} C_{i}=D\left(p^{*}\right) .
$$

The proof is quite technical and is left to the appendix.

The proposition basically states that the only equilibrium is such that demand matches capacity for all providers, all of them setting the same unit price.

Remark 2: Proposition 3 establishes that the demand configuration at the user Wardrop equilibrium corresponding to the competitive Nash equilibrium of the provider pricing game is exactly the demand pointed out in Proposition 1. In other terms, letting providers setting selfishly their prices and users choosing selfishly their provider yields the same social welfare as a perfect coordination mechanism would have given. This suggest that when congestion and competition are sufficient (in order for Assumption B to hold), no regulation schemes are needed since the market itself determines the right prices and allocations.

Remark 3: If Assumption B is not verified, for example if one provider $i$ has a strong power in the sense that $C_{i}>$ $\sum_{j \neq i} C_{j}$, then Proposition 3 does not hold. In that case it is possible that the dominating provider take benefit of its position to improve its revenue by setting a high price $p_{i}>p^{*}$.

\section{GAME ON DECLARED CAPACITIES}

In this section, we assume that a provider $i \in \mathcal{I}$ may voluntarily declare a false value $C_{i}^{n} \leq C_{i}$ of its capacity $C_{i}$. Only the declared values $C_{i}^{n} \leq C_{i}$ are feasible: whereas provider $i$ can easily degrade artificially its service rate, it cannot increase it above its real capacity $C_{i}$, and a false declaration aimed at increasing one's demand to get a larger benefit would consequently be detected.

From the point of view of a provider's net benefit, there are two opposite effects of lowering one's capacity: on the one hand the unit selling price at equilibrium increases and the managing cost decreases because the quantity sold decreases, whereas on the other hand less quantity sold means less revenue. The effect that overcomes the other depends on the elasticity of demand, i.e. the extent to which total demand is affected by variations of unit price. Recall that when the demand function $D$ is differentiable, the elasticity of demand at a unit selling price $p$ is defined by

$$
\frac{p D^{\prime}(p)}{D(p)} \text {. }
$$

Remark that for all $p$, the elasticity at $p$ is a negative number due to the nonincreasingness of $D$.

Since the demand is the inverse function of the marginal valuation $v$, a small demand elasticity (in absolute value) means that $v$ decreases quickly with the unit price. In such a case, a small decrease of total demand corresponds to a large price increase, and the positive effect on revenue of underdeclaring one's capacity exceeds the negative one. The next proposition gives a sufficient condition on demand elasticity for that situation not to occur: if demand is sufficiently elastic (i.e. absolute value of elasticity larger than 1), then providers have interest in truthfully declaring their capacity.

Proposition 4: Under Assumption B, if the absolute value of demand elasticity is larger than 1 for $p \geq p^{*}$, i.e.

$$
\forall p \geq p^{*}, \quad \frac{-p D^{\prime}(p)}{D(p)} \geq 1,
$$

then no provider can increase its revenue by artificially lowering its capacity.

Proof: Without loss of generality, it is sufficient to prove that the net benefit of provider 1 always decreases when it underdeclares its capacity. For any value $C_{1}^{n} \leq C_{1}$ of the declared capacity of provider 1 , Assumption $\mathrm{B}$ still holds with declared capacities, and the equilibrium of the price competition game is therefore given by Proposition 3. If we define $C_{-1}:=\sum_{i \neq 1} C_{i}$, the unit price $p^{* n}$ at the price competition equilibrium is then

$$
p^{* n}=v\left(C_{1}^{n}+C_{-1}\right),
$$

each provider $i \neq 1$ gets demand $C_{i}$, and provider 1 obtains demand $C_{1}^{n}$ and gets total benefit $R_{1}^{n}=C_{1}^{n} p^{* n}-\ell_{1}\left(C_{1}^{n}\right)$. Notice that $p^{* n} \geq p^{*}$ due to the nonincreasingness of the marginal valuation function $v$.

From (13), the total demand should therefore verify

$$
\begin{aligned}
C_{1}^{n}+C_{-1} & =D\left(p^{* n}\right)=D\left(p^{*}\right)+\int_{p^{*}}^{p^{* n}} D^{\prime}(p) d p \\
& \leq D\left(p^{*}\right)-\int_{p^{*}}^{p^{* n}} \frac{D(p)}{p} d p \\
& \leq D\left(p^{*}\right)-C_{-1} \int_{p^{*}}^{p^{* n}} \frac{1}{p} d p,
\end{aligned}
$$

where the second line comes from (12), and the third one from the nonincreasingness of total demand and (13) which imply that $D(p) \geq C_{-1}$ for all $p \in\left[p^{*}, p^{* n}\right]$.

We then obtain

$$
C_{1}^{n} \leq C_{1}-C_{-1} \log \left(p^{* n} / p^{*}\right):=\bar{d}_{1} .
$$


From the convexity of $\ell_{1}$ and Assumption A (implied by Assumption B), we have

$$
R_{1}^{n} \leq p^{* n} \bar{d}_{1}-\ell_{1}\left(\bar{d}_{1}\right) .
$$

By truthfully declaring $C_{1}$, provider 1 would get a net benefit $R_{1}=p^{*} C_{1}-\ell_{1}\left(C_{1}\right)$. The gain of underdeclaring one's capacity can thus be upperbounded:

$$
\begin{aligned}
R_{1}^{n}-R_{1} \leq & p^{* n} \bar{d}_{1}-p^{*} C_{1}+\ell_{1}\left(C_{1}\right)-\ell_{1}\left(\bar{d}_{1}\right) \\
\leq & p^{* n} \bar{d}_{1}-p^{*} C_{1}+\left(1-C_{1} / C_{-1}\right) p^{*}\left(C_{1}-\bar{d}_{1}\right) \\
= & C_{1}\left(p^{* n}-p^{*}\right)+\log \left(p^{* n} / p^{*}\right)\left(p^{*}\left(C_{-1}-C_{1}\right)\right. \\
& \left.-p^{* n} C_{-1}\right) .
\end{aligned}
$$

where the second line stems from the convexity of $\ell_{1}$ and Assumption B, and the third one from (14).

Define $x:=p^{* n} / p^{*} \geq 1$. We can thus write

$$
R_{1}^{n}-R_{1} \leq p^{*} g(x)
$$

where $g: x \mapsto p^{*} C_{1}(x-1)+p^{*} \log (x)\left(\left(C_{-1}-C_{1}\right)-x C_{-1}\right)$. It is then straightforward to check that

- $g$ is concave,

- $g(1)=0$,

- $g$ is derivable and $g^{\prime}(1)=0$.

Those three points imply that $g(x) \leq 0$ for all $x \geq 1$, which from (15) means that $R_{1}^{n} \leq R_{1}$ and gives the proposition.

\section{CONCLUSION}

In this paper, we have analyzed a pricing game among service providers with fixed capacities, such that each provider has an access price it can play with. We have characterized how demand will be naturally split between those providers, following Wardrop's principle, and determined the existence and uniqueness of the Nash equilibrium of the pricing game. We have shown that this equilibrium corresponds to the socially optimal point (meaning that the price of anarchy is 1), and discussed the interest for providers to voluntarily reduce their capacity.

There are different ways to extend the results we have obtained. First of all, we could investigate the case where providers share, at least partially, their capacities: does it lead to a price war? Another interesting issue concerns the capacity expansion game. Indeed, capacity could also be an important parameter providers can play with, at the same time as prices: what would be the resulting equilibrium? Finally, it would be of interest to extend the game to a multiclass system with different priority levels at each provider similarly to what was done in [15] for the monopolistic game.

\section{ACKNOWLEDGMENT}

The authors would like to thank New-Zealand ISAT program for igniting this research activity, and acknowledge the support of Euro-FGI Network of Excellence through the specific research project CAP (Competition Among Providers), as well as WINEM ANR project for the second author.

\section{APPENDIX}

We prove here Proposition 3.

Proof: The proof can be decomposed into two steps:

1) We first show that the point such that $d_{i}=C_{i}$ and $p_{i}=p^{*} \forall i$, with $p^{*}=v\left(\sum_{i \in \mathcal{I}} C_{i}\right)$ defines a Nash equilibrium;

2) then we prove that no other point can be a Nash equilibrium.

Step 1: $p_{i}=p^{*}, \forall i$ is a Nash equilibrium.

Note first that in order to have $R_{i} \geq 0$, i.e., $p_{i} d_{i}-\ell_{i}\left(d_{i}\right) \geq 0$ at the equilibrium point, means that $p C_{i}-\ell_{i}\left(C_{i}\right) \geq 0$, i.e. here

$$
\frac{\ell_{i}\left(C_{i}\right)}{C_{i}} \leq v\left(\sum_{j \in \mathcal{I}} C_{j}\right) .
$$

This last inequality indeed holds under Assumption $\mathrm{A}$ in the realistic case when $\ell_{i}(0)=0$, due to the convexity of $\ell_{i}$ for all $i$.

We begin by establishing that the price configuration with $p_{i}=p^{*}$ for all $i$ where $p^{*}$ is given by (11) is indeed a Nash equilibrium of the pricing game. First notice that in this situation, the common perceived price for users is $p^{*}$, and the demand of each provider is $d_{i}=C_{i}$. We need to prove that in such a price configuration, no provider can improve its revenue by unilaterally changing its suggested price. Without loss of generality, consider a possible move of provider 1 from $p^{*}$ to $p_{1}^{n} \neq p^{*}$. We distinguish two cases.

- If $p_{1}^{n}<p^{*}$ then applying Lemma 1 to a change from $p^{*}$ to $p_{1}^{n}$ we get that

- the new perceived price $\underline{p}^{n}$ at the Wardrop equilibrium is lower than the original perceived price: $\underline{p}^{n} \leq p^{*}$, because of a smaller function $f_{1}$ while the others remain unchanged.

- the new allocation $d_{1}^{n}$ at provider 1 is strictly positive, therefore from Lemma 1 it is strictly above the original one: $d_{1}^{n}>C_{1}$, which implies that $d_{1}^{n}=C_{1} \underline{p}^{n} / p_{1}^{n}$ from (8).

Consequently, the revenue gain of provider 1 for lowering its price is

$$
\begin{aligned}
R_{1}^{n}-R_{1} & =p_{1}^{n} d_{1}^{n}-p^{*} C_{1}+\ell_{1}\left(C_{1}\right)-\ell_{1}\left(d_{1}^{n}\right) \\
& =\underbrace{\left(\underline{p}^{n}-p^{*}\right)}_{<0} C_{1}+\underbrace{\ell_{1}\left(C_{1}\right)-\ell_{1}\left(d_{1}^{n}\right)}_{<0} \\
& <0 .
\end{aligned}
$$

- If $p_{1}^{n}>p^{*}$ then from Lemma 1 we have $\underline{p}^{n} \geq p^{*}$ and $d_{1}^{n}<C_{1}$. First notice that if $p_{1}^{n} \geq \inf \{p: D(p) \leq$ $\left.p \sum_{j \neq 1} C_{j} / p^{*}\right\}$ (i.e. the situation displayed in Figure 2), then $d_{i}^{n}=0$ and provider 1 gets a null profit, that is lower than the profit yielded by playing $p^{*}$ as noticed at the beginning of the proof. Therefore only the case when $d_{1}^{n}>0$ needs to be proved. Actually since $0<d_{1}^{n}<C_{1}$ then we have $\underline{p}=p_{1}^{n}>p^{*}$, which from (8) implies that 


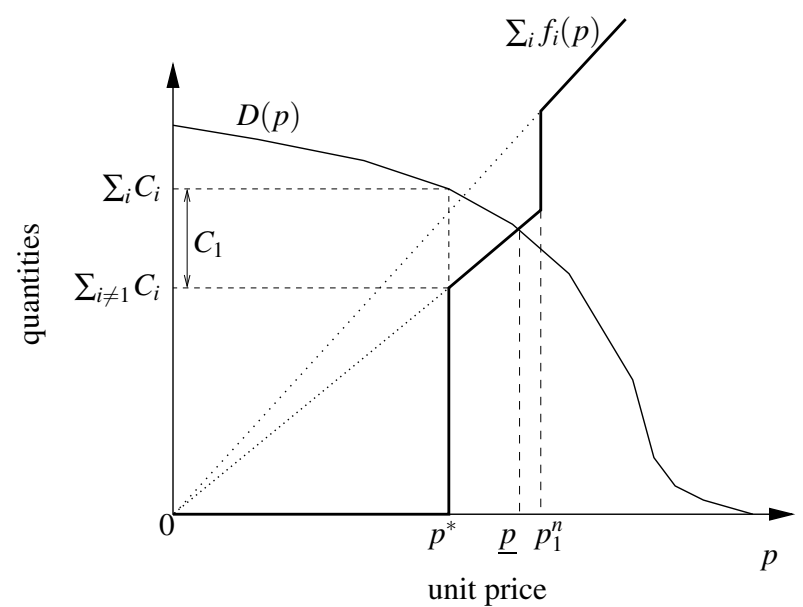

Fig. 2. Wardrop equilibrium if player 1 switches from $p^{*}$ to $p_{1}^{n} \geq \inf \{p$ : $\left.D(p) \leq p \sum_{j \neq 1} C_{j} / p^{*}\right\}$.

$$
\begin{aligned}
& \forall i \neq 1, d_{i}^{n}=C_{i} p_{1}^{n} / p^{*} \text {, and } \\
& \qquad \begin{aligned}
d_{1}^{n} & =D\left(p_{1}^{n}\right)-p_{1}^{n} \sum_{j \neq 1} C_{j} / p^{*} \\
& \leq D\left(p^{*}\right)-p_{1}^{n} \sum_{j \neq 1} C_{j} / p^{*},
\end{aligned}
\end{aligned}
$$

where the first line second line comes from (11) and the nonincreasingness of the demand function. This is illustrated in Figure 3. In that case, the revenue gain for provider 1 is

$$
\begin{aligned}
R_{1}^{n}-R_{1} & =p_{1}^{n} d_{1}^{n}-p^{*} C_{1}+\ell_{1}\left(C_{1}\right)-\ell_{1}\left(d_{1}^{n}\right) \\
& \leq p_{1}^{n} d_{1}^{n}-p^{*} C_{1}+\ell_{1}^{\prime}\left(C_{1}\right)\left(C_{1}-d_{1}^{n}\right) \\
& \leq d_{1}^{n} \underbrace{\left(p_{1}^{n}-p^{*}\left(1-\frac{C_{1}}{\sum_{j \neq 1} C_{j}}\right)\right)}_{>0} \\
& \leq-\frac{\sum^{*} \frac{C_{1}^{2}}{\sum_{j \neq 1} C_{j}}}{p^{*}}\left(p_{1}^{n}-p^{*}\right)^{2} \\
& <0,
\end{aligned}
$$

where the second and third line respectively stem from the convexity of $\ell_{1}$ and Assumption $\mathrm{B}$, and the fourth line is obtained after upper-bounding $d_{1}^{n}$ by the expression in (16) and performing some simplifications.

Step 2: uniqueness of the Nash equilibrium.

Now knowing that there exists a Nash equilibrium under Assumption B, we establish that this equilibrium is unique. Indeed, the proof of the uniqueness only needs Assumption A to hold, as we see below.

To prove uniqueness, consider a Nash equilibrium of the pricing game, i.e. a price configuration, and decompose the set of providers $\mathcal{I}$ into three disjoint subsets: $\mathcal{I}=\mathcal{I}_{s} \cup \mathcal{I}_{0} \cup \mathcal{I}_{u}$,

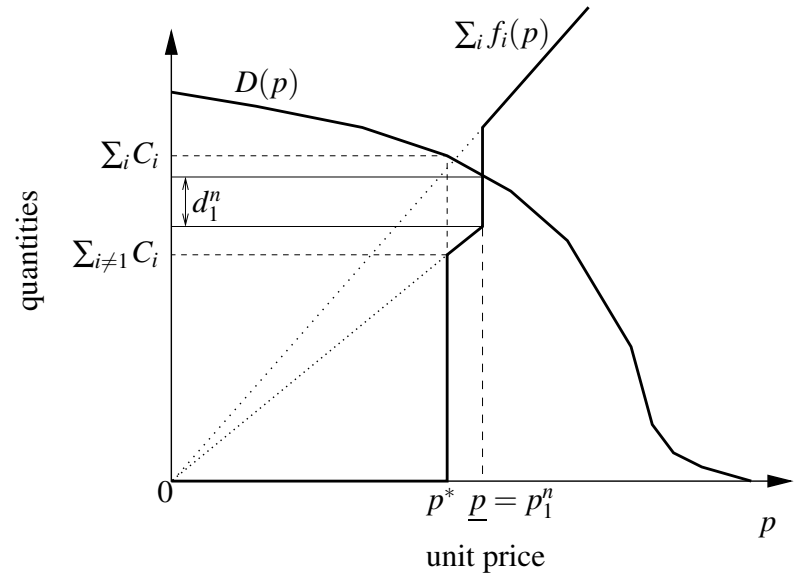

Fig. 3. Wardrop equilibrium if player 1 switches from $p^{*}$ to $p_{1}^{n}<\inf \{p$ : $\left.D(p) \leq p \sum_{j \neq 1} C_{j} / p^{*}\right\}$.

where

$$
\begin{aligned}
& \mathcal{I}_{s}:=\left\{i \in \mathcal{I}: d_{i}>C_{i}\right\}, \\
& \mathcal{I}_{0}:=\left\{i \in \mathcal{I}: d_{i}=C_{i}\right\}, \\
& \mathcal{I}_{u}:=\left\{i \in \mathcal{I}: d_{i}<C_{i}\right\} .
\end{aligned}
$$

It is sufficient to show that $\mathcal{I}_{s}$ and $\mathcal{I}_{u}$ are empty sets, since only the price configuration $\mathbf{p}=(\underline{p}, \ldots, \underline{p})$ can lead to the demand configuration with $d_{i}=C_{i}$ for all $i$.

We first prove that $\mathcal{I}_{s}=\emptyset$. Assume it is not the case, and consider $i_{s} \in \mathcal{I}_{s}$. We study the influence of provider $i_{s}$ increasing its unit price $p_{i_{s}}$ to $p_{i_{s}}^{n}=p_{i_{s}}+\varepsilon$ for $\varepsilon>0$, all other providers keeping their price unchanged. From the continuity of the perceived unit price at Wardrop equilibrium in terms of the price configuration, there exists $\varepsilon>0$ such that $\underline{p}^{n}>p_{i_{s}}^{n}$, which implies that $d_{i_{s}}^{n}=C_{i_{s}} \underline{p}^{n} / p_{i_{s}}^{n}$ from the relation between demand and perceived price. For this $\varepsilon$, the net benefit of provider $i$ is $R_{i_{s}}^{n}=\underline{p}^{n} C_{i_{s}}-\ell_{i_{s}}\left(d_{i_{s}}^{n}\right)$. Its gain in net benefit with respect to the initial situation is therefore

$$
R_{i_{s}}^{n}-R_{i_{s}}=C_{i_{s}}\left(\underline{p}^{n}-\underline{p}\right)+\left(\ell_{i_{s}}\left(d_{i_{s}}\right)-\ell_{i_{s}}\left(d_{i_{s}}^{n}\right)\right)>0,
$$

where the strict positivity comes from Lemma 1 and the strict increasingness of $\ell_{i_{s}}$. We have established that any provider in the set $\mathcal{I}_{s}$ can strictly increase its net benefit by increasing its price, which implies that

$$
\text { at a Nash equilibrium, } \quad \mathcal{I}_{s}=\emptyset \text {. }
$$

Remark that $\mathcal{I}_{s}=\emptyset$ implies that $d_{i} \leq C_{i}$ for all $i \in \mathcal{I}$, thus $d=D(\underline{p}) \leq \sum_{i} C_{i}$ and consequently $\underline{p} \geq v\left(\sum_{i \in \mathcal{I}} C_{i}\right)$.

Now we assume that $\mathcal{I}_{u} \neq \emptyset$, and prove that at least a provider $i_{u} \in \mathcal{I}_{u}$ can strictly increase its net benefit by decreasing its price. We still consider a Nash equilibrium price configuration, and denote by $\underline{p}$ the user perceived price for that price configuration. The total demand should therefore be $D(\underline{p})$. Since we previously proved that $\mathcal{I}_{s}=\emptyset$, then $D(\underline{p})<\sum_{i \in \mathcal{I}} C_{i}=D\left(p^{*}\right)$, with $p^{*}=v\left(\sum_{i \in \mathcal{I}} C_{i}\right)$, therefore

$$
\underline{p}>p^{*} .
$$


When there are at least two providers, at a Nash equilibrium with $\mathcal{I}_{u} \neq \emptyset$ then for all $i$ we have $d_{i} \leq \min \left(C_{i}, D(\underline{p})\right)$, since $\mathcal{I}_{s}=\emptyset$ and total demand equals $D(p)$. Moreover, there necessarily exists a provider $i_{u}$ for which $d_{i}<D(p)$ : indeed there exists at least a provider in $\mathcal{I}_{u}$, and if this provider gets all the demand then it implies that all the other providers have demand 0 . Thus consider $i_{u}$ such that

$$
d_{i_{u}}<\min \left(C_{i_{u}}, D(\underline{p})\right) .
$$

We now prove that provider $i_{u}$ can strictly improve its benefit by changing its price from $p_{i_{u}} \geq \underline{p}$ to $p_{i_{u}}^{\varepsilon}:=\underline{p}-\varepsilon$ for a sufficiently small $\varepsilon>0$. We distinguish two cases.

- If $C_{i_{u}} \leq D(\underline{p})$, then we easily see from (9) that the new perceived price $\underline{p}^{\varepsilon}$ verifies

$$
p_{i_{u}}^{\varepsilon}=\underline{p}-\varepsilon<\underline{p}^{\varepsilon} \leq \underline{p} .
$$

By changing its price to $\underline{p}-\varepsilon$, provider $i_{u}$ is the only provider with the lowest declared unit price, therefore from Definition 2 its demand $d_{i_{u}}^{\varepsilon}$ equals $C_{i_{u}} \frac{\underline{p}^{\varepsilon}}{\underline{p}-\varepsilon}$, which tends to $C_{i_{u}}$ when $\varepsilon$ tends to 0 .

- If $C_{i_{u}}>D(p)$ then for $\varepsilon$ sufficiently small (such that $D(p-\varepsilon) \leq \bar{C}_{i_{u}}$ ), provider $i_{u}$ gets all the demand, i.e. $d_{i_{u}}^{\varepsilon}=D(\underline{p}-\varepsilon)$. When $\varepsilon$ tends to 0 , that demand tends to $D(\underline{p})$ because of the continuity of the demand function.

Consequently, for a sufficiently small $\varepsilon$, the demand for provider $i_{u}$ of switching from price $p_{i_{u}}$ to price $\underline{p}-\varepsilon$ can be arbitrarily close to $y:=\min \left(C_{i_{u}}, D(\underline{p})\right)>d_{i_{u}}$, and the corresponding revenue gain can then be arbitrarily close to

$$
\begin{aligned}
\underline{p}\left(y-d_{i_{u}}\right)-\ell_{i_{u}}(y)+\ell_{i_{u}}\left(d_{i_{u}}\right) & \geq\left(\underline{p}-\ell_{i_{u}}^{\prime}(y)\right) \underbrace{\left(y-d_{i_{u}}\right)}_{>0} \\
& \geq\left(\underline{p}-\ell_{i_{u}}^{\prime}\left(C_{i_{u}}\right)\right)\left(y-d_{i_{u}}\right) \\
& \geq\left(\underline{p}-p^{*}\right)\left(y-d_{i_{u}}\right) \\
& >0,
\end{aligned}
$$

where the first and second lines come from the convexity of $\ell_{i_{u}}$ and $y \leq C_{i_{u}}$, the third one from Assumption $\mathrm{A}$, and the last line stems from (21). Consequently, provider $i_{u}$ can strictly improve its net benefit by unilaterally changing its declared price, which contradicts the Nash equilibrium condition and establishes that we necessarily have

$$
\text { at a Nash equilibrium, } \quad \mathcal{I}_{u}=\emptyset .
$$

Relations (20) and (23) together with the demand relation $\sum_{i \in \mathcal{I}} d_{i}=D(\underline{p})$ imply the uniqueness of the Nash equilibrium: a Nash equilibrium is necessarily such that each provider $i$ declares unit price $p_{i}=p^{*}$.

\section{REFERENCES}

[1] P. B. Key and L. Massoulié, "User policies in a network implementing congestion pricing," in Proc. of Workshop of Internet Service Quality Economics (ISQE). MIT Press, Cambridge, Massachusetts, 1999.

[2] S. Low and D. Lapsley, "Optimization Flow Control, I: Basic Algorithm and Convergence," IEEE/ACM Transactions on Networking, vol. 7, no. 6, 1999.
[3] P. Maillé and B. Tuffin, "Pricing the internet with multibid auctions," IEEE/ACM Transactions on Networking, vol. 14, no. 5, pp. 992-1004, Oct 2006.

[4] H. Yaïche, R. R. Mazumdar, and C. Rosenberg, "A game theoretic framework for bandwidth allocation and pricing in broadband networks," IEEE/ACM Transactions on Networking, vol. 8, no. 5, pp. 667-678, 2000.

[5] F. Kelly, A. Mauloo, and D. Tan, "Rate control in communication networks: shadow prices, proportional fairness and stability," Journal of the Operational Research Society, vol. 49, pp. 237-252, 1998.

[6] R. La and V. Anantharam, "Window-Based Control with Heterogeneous Users," in Proceedings of IEEE INFOCOM 2001, 2001.

[7] T. Başar and R. Srikant, "Revenue-maximizing pricing and capacity expansion in a many-users regime," in Proc. of IEEE INFOCOM, 2002.

[8] E. Campos-Náñez and S. D. Patek, "On-line tuning of prices for network services," in Proc. of IEEE INFOCOM, 2003.

[9] Y. Hayel, D. Ros, and B. Tuffin, "Less-than-Best-Effort Services: Pricing and Scheduling," in IEEE INFOCOM 2004, Hong-Kong, China, March 2004.

[10] I. Paschalidis and J. Tsitsiklis, "Congestion-Dependent Pricing of Network Services," IEEE/ACM Transactions on Networking, vol. 8, no. 2, pp. 171-184, 2000.

[11] M. Osborne and A. Rubenstein, A Course on Game Theory. MIT Press, 1994.

[12] C. Courcoubetis and R. Weber, Pricing Communication NetworksEconomics, Technology and Modelling. Wiley, 2003.

[13] L. DaSilva, "Pricing of QoS-Enabled Networks: A Survey," IEEE Communications Surveys \& Tutorials, vol. 3, no. 2, 2000.

[14] B. Tuffin, "Charging the Internet without bandwidth reservation: an overview and bibliography of mathematical approaches," Journal of Information Science and Engineering, vol. 19, no. 5, pp. 765-786, 2003.

[15] P. Marbach, "Analysis of a static pricing scheme for priority services," IEEE/ACM Transactions on Networking, vol. 12, no. 2, 2004.

[16] R. Gibbens, R. Mason, and R. Steinberg, "Internet service classes under competition," IEEE Journal on Selected Areas in Communications, vol. 18, no. 12, pp. 2490-2498, 2000.

[17] R. El-Azouzi, E. Altman, and L. Wynter, "Telecommunications network equilibrium with price and quality-of-service characteristics," in Proc. of 18th International Teletraffic Congress, Berlin, Germany, Sept 2003.

[18] Z. Liu, L. Wynter, and C. Xia, "Pricing information services in a competitive market: avoiding price wars," INRIA, Tech. Rep., 2002.

[19] O. Ileri, D. Samardzija, T. Sizer, and N. Mandayam, "Demand responsive pricing and competitive spectrum allocation via a spectrum server," in Proc. of IEEE DySpan 2005, 2006.

[20] M. Felegyhazi and J. Hubaux, "Wireless operators in a shared spectrum," in Proc. of IEEE INFOCOM, 2006.

[21] L. He and J. Walrand, "Pricing internet services with multiple providers," in Proc. of the 41st Allerton Conference on Communication, Control and Computing, 2003.

[22] — "Pricing and revenue sharing strategies for internet service providers," in Proc. of IEEE INFOCOM, Mar 2005.

[23] S. Shakkottai and R. Srikant, "Economics of network pricing with multiple ISPs," in Proc. of IEEE INFOCOM 2005, Miami, FL, USA, 2005.

[24] D. Acemoglu and A. Ozdaglar, "Competition and efficiency in congested markets," Mathematics of Operations Research, 2006.

[25] A. Hayrapetyan, E. Tardos, and T. Wexler, "A network pricing game for selfish traffic," in Proc. of IEEE PODC, 2006.

[26] D. Acemoglu and A. Ozdaglar, "Price competition in communication networks," in Proc. of IEEE INFOCOM, 2006.

[27] D. Fudenberg and J. Tirole, Game Theory. MIT Press, Cambridge, Massachusetts, 1991

[28] E. Koutsoupias and C. Papadimitriou, "Worst-case equilibria," in Proc of 16th Annual Symposium on Theoretical Aspects of Computer Science (STACS 1999), vol. 1563 of Lecture Notes in Computer Science, 1999, pp. 404-413.

[29] J. Wardrop, "Some theoretical aspects of road traffic research," proceedings of the Institute of Civil Engineers, vol. 1, pp. 325-378, 1957.

[30] M. Beckmann, C. B. McGuire, and C. B. Winsten, Studies in the economics of transportation. Yale University Press, New Heaven, Connecticut, 1956. 